\title{
Disentangling Substance Use and Disorder: Urgency, But Not Sensation Seeking, Predicts Substance- Related Problems Above and Beyond The Degree of Substance Use.
}

Malin Hildebrandt ( $\square$ malin.hildebrandt@tu-dresden.de )

TU Dresden

Raoul Dieterich

TU Dresden

Tanja Endrass

TU Dresden

Research Article

Keywords: substance use, impulsivity, sensation seeking, dimensional, urgency

Posted Date: November 24th, 2020

DOI: https://doi.org/10.21203/rs.3.rs-108038/v1

License: (-) (i) This work is licensed under a Creative Commons Attribution 4.0 International License. Read Full License 


\section{Abstract}

Background: Substance use disorders are reliably associated with high impulsivity and sensation seeking. Importantly, both precede problematic substance use, implicating them as risk factors. Individuals with substance use disorders show variable degrees of substance use (combined quantity and frequency) and substance-related problems and differ in both aspects from healthy controls. Dimensional research has indicated differential associations of impulsivity-related traits as well as sensation seeking with the degree of substance use and substance-related problems. The current study aimed to clarify whether impulsivityrelated traits and sensation seeking predict substance-related problems above and beyond the degree of substance use and are thus specifically linked to problems, the dimension that characterizes substance use disorders.

Method: We assessed impulsivity-related traits and sensation seeking using self-report, as well as delay discounting, a behavioral indicator of impulsivity, in a sample of 258 substance-using adults.

Results: Sensation seeking and impulsivity-related traits significantly predicted the degree of substance use, with sensation seeking explaining the largest portion of variance. In contrast, self-reported impulsivity, in particular when experiencing negative emotions (urgency), but not sensation seeking or delay discounting, predicted substance-related problems when controlling for the degree of substance use.

Conclusions: This suggests that urgency, but not sensation seeking, may be specifically linked to substance-related problems and thus especially relevant for substance use disorders. Taken together, this study underlines the necessity to assess and control for the degree of substance use in risk factor research concerning substance-related problems. Thus, it may inform future research improving targeted prevention and therapy.

\section{Background}

Substance use disorders (SUDs) are characterized not only by substance use itself but by problems related to substance use (1), such as the inability to control substance intake, or the failure to comply with social responsibilities. Not all frequent substance users develop these problems to the same extent. It is crucial for targeted prevention and treatment to identify factors that specifically influence the development of problems. Most of the literature on SUDs relies on comparisons of individuals diagnosed with SUDs and healthy controls in order to do so. Here, the patients differ from healthy controls in both the degree of substance use (quantity and frequency) and substance-related problems. This hinders evaluating whether the examined potential risk factors are truly associated with the development of substance-related problems, the dimension that is critical for the diagnosis of SUDs. The specific associations of potential risk factors with the degree of substance use and substance-related problems remain unclear. Here, we are proposing a dimensional approach examining the associations of potential risk factors with substancerelated problems while statistically controlling for the degree of substance use. Consequently, we assessed both variables separately and dimensionally in a population of substance users of varying degrees of substance use. Using this approach, we aimed to take a first step in re-evaluating the role of impulsivity- 
related traits as risk factors for SUDs. High levels of impulsivity-related traits are robustly found in individuals with SUDs (2-5). So far, it has never been tested whether this is driven by the association of these factors with the degree of substance use or whether they are, beyond that, incrementally relevant for substance-related problems across different substances. Particularly, controlling for the degree of use has been neglected in previous work.

Overall, impulsivity is robustly associated with SUDs (for a review see e.g. 6). Factor analyses of questionnaires assessing impulsivity repeatedly suggested that it may be divided into four distinct, yet related facets: urgency, (lack of) premeditation, (lack of) perseverance and sensation seeking (UPPS model; $7,8)$. The first three facets conform with a narrow definition of impulsivity, understood as the propensity to act quickly while disregarding long-term negative consequences (9) and we will therefore subsume them as impulsivity-related traits. Sensation seeking does not conform with this definition and has recently been pinpointed as a separate construct (10) that correlates only weakly with impulsivity-related traits (between r $=0.00$ and $r=0.18 ; 7,11)$, suggesting that the tendency to seek excitement is to a large extent independent of the tendency to act rashly.

Longitudinal studies suggest that sensation seeking prospectively predicts the risk to develop SUDs (e.g. 4). Further studies indicate impulsivity-related traits as risk factor for SUDs by showing that impulsivityrelated traits prospectively predict the degree of substance use as well as related problems with similar (12, 13) or higher (14) associations of impulsivity-related traits with problems than with the degree of substance use. Notably, substance use and problems do not prospectively predict impulsivity-related traits (15). One study provides first evidence that a composite score that included impulsivity-related measures, sensation seeking and emotional control prospectively predicts substance-related problems even when controlling for substance use (with a medium effect size; 13).

Research further suggests that impulsivity-related traits as well as sensation seeking are differentially related to the degree of substance use and problems. Sensation seeking and premeditation are associated with the degree of substance use (Lynam \& Miller, 2004; Magid \& Colder, 2007; Miller et al., 2003). A metaanalysis that assigned various measures to the UPPS model found that all traits were similarly related to the degree of use, while urgency was the facet most strongly related to problems (16). Urgency, specifically when experiencing negative emotions, has also been linked to other types of problem behavior (e.g. bulimic symptoms; 17). Moreover, although the evidence is so far restricted to alcohol use, urgency and perseverance were shown to be associated with problems, even when controlling for the degree of substance use (Magid \& Colder, 2007). Importantly, the association of sensation seeking with problems disappeared when the degree of substance use was statistically controlled for. These findings underline that the approach we propose here is suitable for identifying factors specifically associated with substance-related problems.

One widely studied behavioral indicator of impulsivity is delay discounting, the subjective devaluation of rewards based on how far in the future they become available (18). This index of the preference for smaller sooner over larger later rewards is of high conceptual relevance to SUD research, as it closely resembles behavior observed in individuals with SUDs, i.e., choosing immediate drug effects over long-term goals 
(e.g., taking care of family or school/job etc.). Empirically, higher delay discounting in individuals with SUDs (19) and associations of delay discounting with substance use frequency (20) and SUD severity (indicating substance-related problems) are consistently observed (21-23). Delay discounting correlates with premeditation (24-27), but also urgency (25) and sensation seeking (27), but a meta-analysis suggests that these associations are overall rather weak.

The goal of this study was to extend the finding that impulsivity-related traits, but not sensation seeking, predict alcohol-related problems above and beyond the degree of substance use (28) by investigating this relationship in a sample of (poly)substance users. Thereby, we aimed to examine if impulsivity-related traits, but not sensation seeking, may be specifically linked to substance-related problems (and thus truly be relevant for SUDs) across different substances. Furthermore, we assessed delay discounting to examine whether self-reported and behavioral impulsivity-related traits distinctly relate to substance use variables. We expected that impulsivity-related traits (self-report and delay discounting) as well as sensation seeking would predict the degree of substance use, while only impulsivity-related traits (more specifically urgency, perseverance and delay discounting), but not sensation seeking, would predict substance-related problems when controlling for the degree of substance use.

\section{Methods}

\subsection{Participants}

The final sample consisted of 258 participants who indicated that they were using at least one substance at least once a month. See Table 1 for sociodemographic and substance use-related characteristics of participants. Further details are available in the supplementary material. Inclusion criteria were (1) age between 18 and 40 years, (2) native German speakers, (3) no reported use of any substance (except for nicotine) on the day of the survey. We excluded these participants to avoid effects of acute intoxication on questionnaire responses. Participants were recruited mainly through advertisements in clubs, webpages associated with the techno music scene, and counseling centers as well as through postings and flyers. Participants gave informed consent prior to participation. Participants could earn course credit (six participants) or take part in a lottery to win one of three 10 euro vouchers. The study protocol was approved by the local ethics committee (EK 146042019) and followed the guidelines stated by the Declaration of Helsinki.

\subsection{Procedure}

Participants completed an online-survey (approx. 20 minutes) comprising sociodemographic information and the measures described below.

\section{Table 1}

Sociodemographic and substance use-related characteristics of participants 


\begin{tabular}{|c|c|c|c|c|}
\hline \multicolumn{3}{|l|}{ Characteristic } & $N$ & $\%$ \\
\hline \multicolumn{5}{|l|}{ Gender } \\
\hline \multicolumn{3}{|l|}{ female } & 148 & 57.4 \\
\hline \multicolumn{3}{|l|}{ male } & 109 & 42.2 \\
\hline \multicolumn{3}{|l|}{ not specified } & 1 & 0.4 \\
\hline \multicolumn{5}{|l|}{ Highest secondary school degree } \\
\hline \multicolumn{3}{|l|}{ upper } & 226 & 87.6 \\
\hline \multicolumn{3}{|l|}{ intermediate } & 28 & 10.9 \\
\hline \multicolumn{3}{|l|}{ lower } & 2 & 0.8 \\
\hline \multicolumn{5}{|l|}{ Current use of } \\
\hline \multicolumn{3}{|l|}{ alcohol } & 236 & 91.5 \\
\hline \multicolumn{3}{|l|}{ nicotine } & 159 & 61.6 \\
\hline \multicolumn{3}{|l|}{ cannabis } & 117 & 45.3 \\
\hline \multicolumn{3}{|l|}{ stimulants } & 79 & 30.6 \\
\hline \multicolumn{3}{|l|}{ hallucinogenic substances } & 29 & 11.2 \\
\hline \multicolumn{3}{|l|}{ opioids } & 14 & 5.4 \\
\hline \multicolumn{3}{|l|}{ misused medication } & 5 & 1.9 \\
\hline \multicolumn{3}{|l|}{ inhalants } & 4 & 1.6 \\
\hline \multicolumn{3}{|l|}{ other substances } & 3 & 1.2 \\
\hline & M & MD & SD & range \\
\hline age & 26.1 & 25.0 & 5.0 & $18-40$ \\
\hline currently used substance classes & 2.5 & 2.0 & 1.3 & $1-6$ \\
\hline substance classes used lifetime & 4.7 & 5.0 & 2.0 & 1-9 \\
\hline
\end{tabular}

Note. $M$ and $S D$ represent mean and standard deviation, respectively.

2.3 Material

\subsubsection{Substance Use Questionnaire}

This questionnaire measured the frequency and quantity of substance use for the following substance classes from the DSM-5: alcohol, tobacco, cannabis, stimulants, opioids, hallucinogenic substances, 
misused medication, inhalants, other substances. Participants were first asked whether they had ever used any of the substances from the respective substance class. If they answered 'yes', participants indicated, among other variables not relevant here, their average frequency of use within the past three months on a 6-point Likert scale ranging from 1 to 6 (almost daily, 3-4 times a week, 1-2 times a week, 1-2 times a month, less than once a month, never) and the average subjective quantity of use on a standard occasion within the past three months on a 5-point Likert scale ranging from 1 (very little) to 5 (very much)[1]. We computed individual degree of substance use scores for each substance class (product of quantity and frequency values, possible maximum: 30 ) and a total degree of substance use score (sum of the individual degree of substance use scores, possible maximum: 270).

\subsubsection{Substance-Related Problems Questionnaire}

This questionnaire measured substance-related problems operationalized by the number of DSM- 5 SUD symptoms from the A criterion participants reported to have experienced within the past 12 months (range 0 - 11). Questions were based on the German version of the Structured Clinical Interview for DSM-5 Disorders - Clinician Version (29) and adapted to the online application. Participants were instructed to consider all substances when judging whether they had experienced each symptom. Thus, this index does not equal a diagnosis of a specific substance use disorder, but rather an index of problem severity across substances as experienced symptoms may be attributable to different substances.

\subsubsection{UPPS Impulsive Behavior Scale}

This 45-item self-report questionnaire assesses four subscales representing facets of impulsivity as identified by Whiteside and Lynam (30): (1) negative urgency (e.g., "In the heat of an argument, I will often say things that I later regret."; possible range: 12 - 48), (2) (lack of ) premeditation (e.g., "I usually make up my mind through careful reasoning."; possible range: 11 - 44), (3) (lack of) perseverance (e.g., "Once I start a project, I almost always finish it."; possible range: 10 - 40), and (4) sensation seeking (e.g., "I welcome new and exciting experiences and sensations, even if they are a little frightening and unconventional."; possible range: 11 - 44). Items are rated on a 4-point Likert scale ranging from 1 (strongly agree) to 4 (strongly disagree). If necessary, items are reverse coded so that higher values on a subscale always indicate higher impulsivity/sensation seeking. The subscales of the German version (31) have good internal consistency (cronbachs $a=.81-.85$ ) and external validity (32).

\subsubsection{Monetary Choice Questionnaire (MCQ)}

The MCQ (German version; 33) assesses delay discounting. It comprises 27 binary choices between varying amounts of money (8 - 66 Euros) available either now or with a delay (7-238 days). Values and delays of the options are predefined to cover a high range of discounting rates for low, medium, and high values of delayed rewards, respectively. The discounting rate $k$ can be modeled assuming a hyperbolic function (34)

[1] For reasons of brevity of the online survey and our decision to combine individual substances to substance classes, we did not assess objective quantities of substance use. The results of this study do 
not change when using only total frequency rather than the total degree of substance use score as a control variable (see supplementary material for further details).

$$
V=\frac{A}{1+k D}
$$

where $V$ is the subjective value of a delayed reward with the amount $A$ after the delay $D$.

The parameter $k$ is estimated based on the proportion of choices that are consistent with every possible value of $k$ (for a thorouh description please refer to 35). As $k$ is dependent on value (36), $k$ is estimated for small, medium and large delayed rewards, respectively, and the geometric mean of these values constitutes an individual's $k$-value.

\subsection{Data analysis}

All analyses were conducted in R (37). We log-transformed the $k$-parameter due to skewedness. Pearson's correlations were computed to examine bivariate associations. Two-tailed tests of the difference between two dependent correlations with one variable in common were conducted to test whether correlations differed significantly. Subsequently, we computed partial correlations of all predictor variables with substance-related problems controlled for the degree of substance use to explore their respective contribution outside the context of other predictor variables. Last, we conducted two hierarchical linear regressions for the degree of substance use and substance-related problems to identify the unique variance explained by sensation seeking, impulsivity-related traits, and $\log (k)$. For both regression models, we entered gender as a covariate in the first step, because it was significantly related to both outcome variables, while age was not ( $p s>.05$ ). We excluded the group "gender not specified" from these regression analyses $(n=1)$. For the model predicting the degree of substance use, we added the UPPS subscales in the second and $\log (k)$ in the third step. For the model predicting substance-related problems, we added the degree of substance use as a covariate in the second step, the UPPS subscales in the third step and $\log (k)$ in the fourth step. To test on an exploratory basis whether a regressor explained more variance in the criterion variable than another regressor, we computed finite-sample F-statistics using the "linearHypothesis" function from the car package (38). We applied a significance level of $a=.05$ for all analyses.

\section{Results}

\subsection{Bivariate correlations}

Discounting rates ranged from 0.000158 to 0.24853 , with some choosing exclusively the smaller sooner or the larger later option, and were not significantly associated with any of the UPPS subscales (all $p s>.05$ ), except for a small positive correlation with urgency (see Table 2 for an overview of correlations). Delay discounting and (lack of) premeditation were significantly, but weakly, correlated with both the degree of substance use and problems. (Lack of) perseverance displayed stronger correlations (small to moderate) 
with both substance use variables. Sensation seeking correlated significantly stronger with the degree of substance use than with problems, $r=.38$ vs. $r=.23, t(256)=2.72, p=.01$. In turn, urgency correlated significantly stronger with substance-related problems than with the degree of substance use, $r=.33, v s . r=$ $.18, t(256)=2.67, p=.01$. The degree of substance use and substance-related problems were strongly correlated.

\section{Table 2}

Means, Standard Deviations, and Correlations with Confidence Intervals for UPPS subscales, Delay Discounting $(\log (k))$, the Degree of Substance Use and Substance-Related Problems

\begin{tabular}{|c|c|c|c|c|c|c|c|c|c|}
\hline & Variable & $M$ & $S D$ & 1 & 2 & 3 & 4 & 5 & 6 \\
\hline & \multicolumn{9}{|l|}{ UPPS } \\
\hline 1. & Urgency & 26.77 & 5.96 & & & & & & \\
\hline \multirow[t]{2}{*}{2.} & Premeditation & 23.59 & 4.50 & $.32 * \star$ & & & & & \\
\hline & & & & $\begin{array}{l}{[.20} \\
.42]\end{array}$ & & & & & \\
\hline \multirow[t]{2}{*}{3.} & Persistence & 20.40 & 4.41 & $.40 * \star$ & $.26 * \star$ & & & & \\
\hline & & & & $\begin{array}{l}{[.29} \\
.50]\end{array}$ & $\begin{array}{l}{[.14} \\
.37]\end{array}$ & & & & \\
\hline \multirow[t]{3}{*}{4.} & $\begin{array}{l}\text { Sensation } \\
\text { Seeking }\end{array}$ & 32.00 & 7.26 & -.00 & $.32 \star \star$ & .01 & & & \\
\hline & & & & $\begin{array}{l}{[-.12,} \\
.12]\end{array}$ & $\begin{array}{l}{[.21} \\
.43]\end{array}$ & $\begin{array}{l}{[-.12,} \\
.13]\end{array}$ & & & \\
\hline & MCQ & & & & & & & & \\
\hline \multirow[t]{3}{*}{5.} & $\log (k)$ & -2.28 & 0.75 & $.14^{\star}$ & -.00 & .04 & -.01 & & \\
\hline & & & & $\begin{array}{l}{[.01} \\
.25]\end{array}$ & $\begin{array}{l}{[-.12,} \\
.12]\end{array}$ & $\begin{array}{l}{[-.08,} \\
.16]\end{array}$ & $\begin{array}{l}{[-.14,} \\
.11]\end{array}$ & & \\
\hline & Substance use & iables & & & & & & & \\
\hline \multirow[t]{2}{*}{6.} & Degree of use & 35.89 & 19.36 & $.18^{\star \star}$ & $.16^{*}$ & $.22^{\star \star}$ & $.38^{\star *}$ & $.18^{\star *}$ & \\
\hline & & & & $\begin{array}{l}{[.05} \\
.29]\end{array}$ & $\begin{array}{l}{[.04} \\
.28]\end{array}$ & $\begin{array}{l}{[.10,} \\
.34]\end{array}$ & $\begin{array}{l}{[.27} \\
.48]\end{array}$ & $\begin{array}{l}{[.06} \\
.30]\end{array}$ & \\
\hline \multirow[t]{2}{*}{7.} & Problems & 4.15 & 2.85 & $.33^{\star \star}$ & $.13^{\star}$ & $.29 \star \star$ & $.23^{\star \star}$ & $.15^{\star}$ & $.55^{\star \star}$ \\
\hline & & & & $\begin{array}{l}{[.22,} \\
.43]\end{array}$ & $\begin{array}{l}{[.01} \\
.25]\end{array}$ & $\begin{array}{l}{[.17,} \\
.39]^{\prime}\end{array}$ & $\begin{array}{l}{[.11} \\
.35]\end{array}$ & $\begin{array}{l}{[.03,} \\
.27]\end{array}$ & $\begin{array}{l}{[.46,} \\
.63]\end{array}$ \\
\hline
\end{tabular}

Note. $M$ and $S D$ represent mean and standard deviation, respectively. Values in square brackets indicate the 95\% confidence interval. UPPS = Urgency Premeditation Perseverance Sensation Seeking Impulsive 
Behavior Scale, MCQ = Monetary Choice Questionnaire, $k=$ delay discounting. ${ }^{\star} p<.05 .{ }^{\star *} p<.01$.

\subsection{Partial correlations}

When controlling for the degree of substance use, urgency $(r(256)=.28, p<.001)$ and perseverance $(r(256)=$ $.20, p=.001)$ significantly correlated with substance-related problems, while premeditation, sensation seeking and $\log (k)$ did not (all $p s>.05)$.

3.3 Relative contributions of UPPS subscales and delay discounting to substance use outcomes

\subsubsection{The degree of substance use}

In the first model, we regressed the degree of substance use on the UPPS subscales and $\log (k)$ while controlling for gender (see Table 3). The hierarchical multiple regression revealed that all UPPS subscales (except for premeditation) as well as $\log (k)$ explained significant unique variance in the degree of substance use, such that higher levels of urgency, perseverance, sensation seeking and delay discounting were associated with more substance use. In the complete model, sensation seeking had a significantly stronger effect on the degree of substance use than all other impulsivity-related predictors respectively, all Fs $>4.13$, all $p s<.05$. The complete model accounted for 25 percent of the variance in the degree of substance use.

\subsubsection{Substance-related problems}

In the second model, we regressed substance-related problems on the UPPS subscales and $\log (k)$ while controlling for age and the degree of substance use (see Table 3). The hierarchical multiple regression revealed that the UPPS urgency subscale explained significant unique variance in substance-related problems, such that higher levels of urgency were associated with more problems above and beyond the degree of substance use, which also predicted substance-related problems. None of the other predictors were significantly associated with substance-related problems. In the complete model, urgency had a significantly stronger effect on substance-related problems than premeditation and $\log (\mathrm{k})$ (all $F \mathrm{~s}>5.57$, all $p s>.05)$ and sensation seeking, but this difference was only a marginally significant $(F=2.96, p=.09)$. The complete model accounted for 39 percent of the variance in substance-related problems.

\section{Table 3}

Hierarchical Multiple Regression Analyses with the UPPS Subscales and Delay Discounting predicting the Degree of Substance Use and Substance-Related Problems 


\begin{tabular}{|c|c|c|c|c|c|c|c|c|c|c|}
\hline \multirow[t]{2}{*}{ Predictors } & \multicolumn{5}{|c|}{ Degree of substance use } & \multicolumn{5}{|c|}{ Substance-related problems } \\
\hline & $\Delta \mathrm{R}^{2}$ & $\mathrm{~F}$ & B & SE & $\beta$ & $\Delta \mathrm{R}^{2}$ & $\mathrm{~F}$ & B & SE & $\beta$ \\
\hline Step 1 & $.087 \star \star$ & 24.45 & & & & $.017 *$ & 4.432 & 4 & & \\
\hline Male gender & & & 11.57 & 2.34 & $.30 \star \star$ & & & 0.75 & 0.35 & $.13^{\star}$ \\
\hline Step 2 & & & & & & $.305^{\star \star}$ & 114.22 & & & \\
\hline Male gender & & & & & & & & -0.23 & 0.31 & -.04 \\
\hline $\begin{array}{l}\text { Degree of } \\
\text { substance } \\
\text { use }\end{array}$ & & & & & & & & 0.08 & 0.01 & $.58^{\star *}$ \\
\hline Step 3 - UPPS & $.144^{\star \star}$ & 11.70 & & & & $.068^{\star *}$ & 6.99 & & & \\
\hline Male gender & & & 7.38 & 2.52 & $.19 \star \star$ & & & -0.06 & 0.33 & -.01 \\
\hline $\begin{array}{l}\text { Degree of } \\
\text { substance } \\
\text { use }\end{array}$ & & & & & & & & 0.07 & 0.01 & $.49 * *$ \\
\hline Urgency & & & 0.52 & 0.21 & $.16^{\star}$ & & & 0.11 & 0.03 & $.23^{\star \star}$ \\
\hline Premeditation & & & -0.09 & 0.28 & -.02 & & & -0.04 & 0.04 & -.06 \\
\hline Perseverance & & & 0.64 & 0.28 & $.15^{\star}$ & & & 0.06 & 0.04 & .10 \\
\hline $\begin{array}{l}\text { Sensation } \\
\text { seeking }\end{array}$ & & & 0.83 & 0.18 & $.31^{\star \star}$ & & & 0.03 & 0.02 & .07 \\
\hline Step 4 - MCQ & $.022^{\star \star}$ & 7.26 & & & & .001 & 0.30 & & & \\
\hline Male gender & & & 6.40 & 2.51 & $.16^{\star}$ & & & -0.08 & 0.34 & -.01 \\
\hline $\begin{array}{l}\text { Degree of } \\
\text { substance } \\
\text { use }\end{array}$ & & & & & & & & 0.07 & 0.01 & $.48^{\star \star}$ \\
\hline Urgency & & & 0.43 & 0.21 & $.13^{*}$ & & & 0.11 & 0.03 & $.22^{\star \star}$ \\
\hline Premeditation & & & -0.08 & 0.27 & -.02 & & & -0.04 & 0.04 & -.06 \\
\hline Perseverance & & & 0.67 & 0.27 & $.15^{\star}$ & & & 0.07 & 0.04 & .10 \\
\hline $\begin{array}{l}\text { Sensation } \\
\text { seeking }\end{array}$ & & & 0.86 & 0.17 & $.32^{\star \star}$ & & & 0.03 & 0.02 & .08 \\
\hline $\log (k)$ & & & 3.88 & 1.44 & $.15^{\star \star}$ & & & 0.10 & 0.19 & .03 \\
\hline Total $\mathrm{R}^{2}$ & $.253^{\star \star}$ & 14.08 & & & & $.391^{\star \star}$ & 22.83 & & & \\
\hline
\end{tabular}


Note. $\Delta R^{2}=$ change in proportion of explained variance; $\mathrm{B}=$ unstandardized estimate; $\mathrm{SE}=$ standard error of $B, \beta=$ standardized estimate. * $p<.05 .{ }^{* \star} p<.01$.

\section{Discussion}

This study examined the differential associations of impulsivity-related traits, sensation seeking, and delay discounting with the degree of substance use (combined quantity and frequency) and substance-related problems in a sample of (poly-)substance users. Thereby, we aimed to disentangle which of these potential risk factors were associated with the degree of substance use and which ones were specifically relevant for substance-related problems and thus for the diagnosis of SUDs.

First, sensation seeking, self-reported urgency and perseverance, as well as delay discounting, explained unique variance in the degree of substance use. Sensation seeking was the strongest predictor, explaining significantly more variance in the degree of substance use than all other predictors. This conforms with the reviewed literature $(8,28,39)$ and indicates that high expressions of both impulsivity and sensation seeking independently, and more so combined, may increase the risk to engage in pronounced substance use. This may already be harmful, e.g. in terms of bodily consequences, but does not necessarily go along with substance-related problems.

Second, the impulsivity-related trait of negative urgency explained significant unique variance in substance-related problems above and beyond the degree of substance use, while sensation seeking did not. This finding is in line with prior research (28) but extends the evidence beyond alcohol use and shows that urgency is specifically linked to substance-related problems, the dimension critical for SUDs, even when controlling for substance use and across substances. Building on evidence showing that high levels of urgency precede problematic substance use (e.g. 39), this finding underlines the role of urgency as a potential general risk factor for substance-related problems. Furthermore, this is the first study to combine self-report with behavioral measures of impulsivity using the approach to SUD risk factor research we propose here. This highlights the special role of urgency for SUDs, as this trait was associated with problems even when controlling for the degree of use as well as behavioral impulsivity (delay discounting) and other impulsivity-related traits.

Delay discounting, a behavioral measure of impulsivity, was weakly associated with both the degree of substance use and problems, but the association with problems disappeared when controlling for the degree of use. This is not surprising, considering that delay discounting was only weakly related to substance-related problems in the first place, closely resembling the effect size found in a meta-analysis (21). This finding indicates that the consistent associations of delay discounting and SUDs described in the literature may be best explained by underlying and confounding differences in the degree of substance use. Although a high degree of use may be inherently harmful, this contradicts the role of delay discounting as a specific risk factor for substance-related problems and thus SUDs discussed in the literature (e.g. 3). However, as this is the first study to examine associations of delay discounting with substance-related problems controlling for the degree of use, this finding needs replication before drawing final conclusions. 
The dimensional approach we applied in this study proved suitable for identifying differential associations of potential SUD risk factors with substance-related problems controlling for the degree of use.

Nonetheless, it requires a longitudinal approach to test whether these factors truly constitute specific risk factors for developing substance-related problems. Furthermore, the delay discounting questionnaire we used in this study uses relatively low monetary rewards. Research suggests that high monetary offers better distinguish substance users from controls (40). Hence, tasks using higher monetary offers may be more suitable for substance-using populations and delay discounting assessed with such tasks may show a specific association with substance-related problems. Importantly, our sample was predominantly highly educated. It is possible that this acted as a protective factor buffering effects of e.g. delay discounting on substance-related problems. Thus, further studies including more representative samples are warranted to consolidate the generalizability of the associations described in this study. Due to temporal restraints in the online-survey, we did not assess substance-related problems separately for each substance or substance class. Therefore, we could not directly test whether the examined associations differed between substances but rather demonstrated that they were overall significant when all substances were combined. Also, the assessment of substance-related problems was purely based on self-report, while clinical interviews may provide a more nuanced and comparable picture. Future studies may include positive urgency, as this facet shows unique associations with substance use outcomes (17) and may thus contribute additional insights.

\section{Conclusion}

This study showed that the impulsivity-related traits of urgency and to some extent perseverance, but not sensation seeking, were specifically linked to substance-related problems, the dimension that is crucial for SUDs. We applied a dimensional approach to SUD risk factor research examining the associations of potential risk factors with substance-related problems while statistically controlling for the degree of substance use. The present study demonstrated that this approach is suitable for identifying factors specifically linked to substance-related problems and thus SUDs. Hence, future longitudinal studies using this approach may clarify the role of impulsivity-related traits, alongside others, as risk factors for SUDs. To identify risk factors specifically linked to the development of substance-related problems, rather than predominantly a high degree of use, would convey important implications for practice. A focus on these factors may help to identify individuals within substance using populations who are more in need for preventive measures in order to avoid a transition to problematic substance use. However, longitudinal studies are needed to substantiate the current finding. Furthermore, they may be relevant target points for therapeutic interventions. Taken together, the approach applied in this study may pose a starting point for further research advancing targeted prevention and therapy.

\section{Declarations}

\section{Ethical approval and consent to participate}


The study protocol was approved by the local ethics committee (Ethikkommission an der Technischen Universität Dresden; EK 146042019) and followed the guidelines stated by the Declaration of Helsinki. Participants were thoroughly informed and indicated consent to participate (informed consent) by a button press in the beginning of the online survey.

\section{Consent for publication}

Not applicable.

\section{Availability of data and materials}

The dataset supporting the conclusions of this article is available in the OSF repository, DOI 10.17605/OSF.IO/CWNRG, https://mfr.de-1.osf.io/render?url=https://osf.io/8wgts/? direct $\% 26$ mode=render\%26action=download\%26mode=render.

\section{Competing interests}

The authors declare that they have no competing interests.

\section{Funding}

This work was supported by a PhD scholarship for Malin Hildebrandt from the Claussen-Simon Stiftung and by the German Research Foundation (Deutsche Forschungsgemeinschaft, grant numbers TRR 265 B01 and EN 906/6). The funding sources had no role in the study design, collection, analysis or interpretation of the data, writing the manuscript, or the decision to submit the paper for publication.

\section{Authors' contributions}

M.H.: Conceptualization, Methodology, Investigation, Formal Analysis, Writing - Original Draft, Project Administration, Funding Acquisition. R. D.: Conceptualization, Methodology, Writing - Review and Editing, Supervision. T. E.: Conceptualization, Resources, Methodology, Writing - Review and Editing, Funding Acquisition, Supervision. All authors contributed to and have approved the final manuscript.

\section{Acknowledgements}

Not applicable.

\section{References}

1. Diagnostic and statistical manual of mental disorders: DSM-5. American Psychiatric A, American Psychiatric Association DSMTF, editors. Arlington, VA: American Psychiatric Association; 2013.

2. Verdejo-García A, Lawrence AJ, Clark L. Impulsivity as a vulnerability marker for substance-use disorders: Review of findings from high-risk research, problem gamblers and genetic association studies. Neuroscience \& Biobehavioral Reviews. 2008;32(4):777-810. 
3. Poulton A, Hester R. Transition to substance use disorders: Impulsivity for reward and learning from reward. Social Cognitive and Affective Neuroscience. 2019:1-10.

4. Foulds JA, Boden JM, Newton-Howes GM, Mulder RT, Horwood LJ. The role of novelty seeking as a predictor of substance use disorder outcomes in early adulthood. Addiction. 2017;112(9):1629-37.

5. Norbury A, Husain M. Sensation-seeking: Dopaminergic modulation and risk for psychopathology. Behavioural Brain Research. 2015;288:79-93.

6. Kozak K, Lucatch AM, Lowe DJE, Balodis IM, MacKillop J, George TP. The neurobiology of impulsivity and substance use disorders: Implications for treatment. Annals of the New York Academy of Sciences. 2019;1451(1):71-91.

7. Whiteside SP, Lynam DR. The Five Factor Model and impulsivity: Using a structural model of personality to understand impulsivity. Personality and Individual Differences. 2001;30(4):669-89.

8. Miller J, Flory K, Lynam D, Leukefeld C. A test of the four-factor model of impulsivity-related traits. Personality and Individual Differences. 2003;34(8):1403-18.

9. Magid V, Maclean MG, Colder CR. Differentiating between sensation seeking and impulsivity through their mediated relations with alcohol use and problems. Addictive behaviors. 2007;32(10):2046-61 .

10. Mitchell M, Potenza M. Addictions and Personality Traits: Impulsivity and Related Constructs. Current Behavioral Neuroscience Reports. 2014;1.

11. Duckworth AL, Kern ML. A meta-analysis of the convergent validity of self-control measures. Journal of Research in Personality. 2011;45(3):259-68.

12. McAdams KK, Donnellan MB. Facets of personality and drinking in first-year college students. Personality and Individual Differences. 2009;46(2):207-12.

13. Mason WA, Toumbourou JW, HerrenkohI TI, Hemphill SA, Catalano RF, Patton GC. Early age alcohol use and later alcohol problems in adolescents: Individual and peer mediators in a bi-national study. Psychology of Addictive Behaviors. 2011;25(4):625-33.

14. Castellanos-Ryan N, Parent S, Vitaro F, Tremblay RE, Séguin JR. Pubertal development, personality, and substance use: a 10-year longitudinal study from childhood to adolescence. J Abnorm Psychol. 2013;122(3):782-96.

15. Fernie G, Peeters M, Gullo MJ, Christiansen P, Cole JC, Sumnall H, et al. Multiple behavioural impulsivity tasks predict prospective alcohol involvement in adolescents. Addiction. 2013;108(11):1916-23.

16. Coskunpinar A, Dir AL, Cyders MA. Multidimensionality in impulsivity and alcohol use: A meta-analysis using the UPPS model of impulsivity. Alcohol Clin Exp Res. 2013;37(9):1441-50.

17. Cyders M, Smith G. Emotion-based dispositions to rash action: Positive and negative urgency. Psych Bull. 2008;134:807-28.

18. Mackillop J, Amlung MT, Few LR, Ray LA, Sweet LH, Munafò MR. Delayed reward discounting and addictive behavior: a meta-analysis. Psychopharmacology (Berl). 2011;216(3):305-21.

19. Wesley MJ, Lohrenz T, Koffarnus MN, McClure SM, De La Garza R, 2nd, Salas R, et al. Choosing money over drugs: The neural underpinnings of difficult choice in chronic cocaine users. Journal of Addiction. 
2014;2014:189853-.

20. Sofis MJ, Budney AJ, Stanger C, Knapp AA, Borodovsky JT. Greater delay discounting and cannabis coping motives are associated with more frequent cannabis use in a large sample of adult cannabis users. Drug and Alcohol Dependence. 2020;207:107820.

21. Amlung M, Vedelago L, Acker J, Balodis I, MacKillop J. Steep delay discounting and addictive behavior: A meta-analysis of continuous associations. Addiction. 2017;112(1):51-62.

22. Claus ED, Kiehl KA, Hutchison KE. Neural and behavioral mechanisms of impulsive choice in alcohol use disorder. Alcohol Clin Exp Res. 2011;35(7):1209-19.

23. Lim AC, Cservenka A, Ray LA. Effects of Alcohol Dependence Severity on Neural Correlates of Delay Discounting. Alcohol and Alcoholism. 2017;52(4):506-15.

24. Lynam DR, Miller JD. Personality Pathways to Impulsive Behavior and Their Relations to Deviance: Results from Three Samples. Journal of Quantitative Criminology. 2004;20(4):319-41.

25. Steward T, Mestre-Bach G, Fernández-Aranda F, Granero R, Perales JC, Navas JF, et al. Delay discounting and impulsivity traits in young and older gambling disorder patients. Addictive Behaviors. 2017;71:96-103.

26. Murphy C, Mackillop J. Living in the here and now: interrelationships between impulsivity, mindfulness, and alcohol misuse. Psychopharmacology (Berl). 2012;219(2):527-36.

27. Cyders MA, Coskunpinar A. Measurement of constructs using self-report and behavioral lab tasks: Is there overlap in nomothetic span and construct representation for impulsivity? Clinical Psychology Review. 2011;31(6):965-82.

28. Magid V, Colder CR. The UPPS Impulsive Behavior Scale: Factor structure and associations with college drinking. Personality and Individual Differences. 2007;43(7):1927-37.

29. Beesdo-Baum K, Zaudig M, First MB, Wittchen HU. SCID-5-CV: Strukturiertes Klinisches Interview für DSM-5-Störungen - Klinische Version : Deutsche Bearbeitung des Structured Clinical Interview for DSM5 Disorders - Clinician Version von Michael B. First, Janet B.W. Williams, Rhonda S. Karg, Robert L. Spitzer: Hogrefe; 2019.

30. Whiteside S, Lynam D, Miller J, Reynolds S. Validation of the UPPS impulsive behavior scale: A fourfactor model of impulsivity. European Journal of Personality. 2005;19:559-74.

31. Schmidt R, Gay P, D'Acremont M, Van der Linden M. A german adaptation of the UPPS impulsive behavior scale: Psychometric properties and factor structure. Swiss Journal of Psychology. 2008;67:107-12.

32. Kämpfe-Hargrave N, Mitte K. A German Validation of the UPPS Impulsive Behavior Scale. European Journal of Psychological Assessment. 2009;25:252-9.

33. Forstmeier S, Maercker A. Selbstkontrolle im höheren Erwachsenenalter: Eine deutsche Version des Delay Discounting Tests von Kirby. Psychotherapie, Psychosomatik, Medizinische Psychologie. 2011;61(06):e19-e33.

34. Ballard K, Knutson B. Dissociable neural representations of future reward magnitude and delay during temporal discounting. Neuroimage. 2009;45(1):143-50. 
35. Gray JC, Amlung MT, Palmer AA, MacKillop J. Syntax for calculation of discounting indices from the monetary choice questionnaire and probability discounting questionnaire. Journal of the Experimental Analysis of Behavior. 2016;106(2):156-63.

36. Kirby KN, Marakovic NN. Delay-discounting probabilistic rewards: Rates decrease as amounts increase. Psychonomic Bulletin \& Review. 1996;3(1):100-4.

37. R Development Core Team. R: A Language and Environment for Statistical Computing. Vienna, Austria: R Foundation for Statistical Computing; 2010.

38. Fox J, Weisberg S. An $\{R\}$ Companion to Applied Regression. Third ed. Thousand Oaks $\{C A\}$ : Sage; 2019.

39. Cyders MA, Flory K, Rainer S, Smith GT. The role of personality dispositions to risky behavior in predicting first-year college drinking. Addiction. 2009;104(2):193-202.

40. Mellis A, Woodford A, Stein J, Bickel W. A second type of magnitude effect: Reinforcer magnitude differentiates delay discounting between substance users and controls: Differences in discounting are magnitude dependent. Journal of the Experimental Analysis of Behavior. 2017;107:151-60.

\section{Supplementary Files}

This is a list of supplementary files associated with this preprint. Click to download.

- HildebrandtSupplements.docx

- HildebrandtSupplements.docx 